\title{
A Comparative Research on Condenser Fault Diagnosis Based on Three Different Algorithms
}

\author{
Xia Fei ${ }^{1,2, *}$, Hao Shuotao ${ }^{2,3}$, Zhang Hao ${ }^{1,2}$ and Peng Daogang ${ }^{2,3}$ \\ ${ }^{I}$ CIMS Center, Tongji University, Shanghai, 201804, P.R. China \\ ${ }^{2}$ College of Automation Engineering, Shanghai University of Electric Power, Shanghai, 200090, P.R. China \\ ${ }^{3}$ Shanghai Key Laboratory of Power Station Automation Technology, Shanghai, 200090, P.R. China
}

\begin{abstract}
In view of artificial neural network, there are some deficiencies in condenser fault diagnosis. The BP neural network used for condenser fault diagnosis is highly nonlinear pattern recognition and high precision of fault diagnosis. The PSO-BP neural network can effectively solve the problem of BP neural network that training time is long and training process is easy to fall into the local minimum. The training results of PSO-BP network in convergence speed and convergence effect are significantly improved. Under the condition of small samples, the calculation results of SVM method are better than the calculation result of the other two methods. Although the recognition ratio of improved PSO-BP(2) and SVM is the same, training time of improved PSO-BP(2) is longer than training time of SVM. The generalization ability of SVM is stronger, and the efficiency of SVM is higher than the neural network. With MATLAB programming, three different algorithms, which are BP neural network, PSO-BP neural network and SVM, are studied and compared for the performance of condenser fault diagnosis. In the models of this study, the research results show that condenser fault diagnosis based on SVM has the fastest convergence speed and the best accuracy.
\end{abstract}

Keywords: Algorithms, BP neural network, Condenser fault diagnosis, PSO-BP neural network, SVM.

\section{INTRODUCTION}

As an important auxiliary equipment of steam turbine generator, condenser has extremely important position in the thermal power generation. But due to a variety of reasons, such as design, installation, overhaul, operating mechanism and so on, the condenser in the operating process will appear all sorts of trouble, especially for low vacuum fault. If the vacuum in condenser is too low, it not only causes the reduce of the effective enthalpy drop of steam in the unit, the drop of circulation heat enthalpy, but also can lead to the rise of the exhaust temperature of the turbine, the deformation and vibration of the row cylinder or other malfunctions [1]. Therefore, the performance of the condenser directly affects the whole economy and safety of the steam turbine unit, and the research of condenser fault diagnosis has an important significance.

In recent years, the artificial neural network is one of the most widely used means in condenser fault diagnosis. Neural network has the advantages of simple structure and strong ability to solve the problem. Therefore, it is the main method for fault diagnosis. But it has problems of local optimization, convergence, long training time, prone to over fitting [2].
The main reason of the above problems is that the neural network pays attention to minimize the error of training samples, while ignoring the confidence range, thus greatly limits the improvement of generalization capability [8]. To overcome the disadvantages of the artificial neural network, the PSO-BP neural network algorithm is proposed. The training results of PSO-BP in convergence speed and convergence effect are significantly improved, and PSO-BP neural network can effectively solve the problem of BP neural network that training time is long and training process is easy to fall into the local minimum [3]. SVM algorithm is proposed. Using SVM well solve the problem of small samples and the classification problem, which can the inherent through methods such as neural network learning and owe learning problems, and SVM also has a strong ability of nonlinear classification [4]. It based on the advantages of the above three algorithms, and applies them to the condenser fault diagnosis.

In order to select the appropriate algorithm and effectively improve the ability of condenser fault diagnosis, this article uses respectively three different algorithms, the BP neural network, PSO-BP neural network and SVM, to diagnosis condenser fault through the establishment of the condenser knowledge base [5]. The convergence speed and the accuracy of the results are analyzed and compared. 
Table 1. The condenser fault symptoms set.

\begin{tabular}{|c|c|c|c|}
\hline Symbol & Fault Symptom & Symbol & Fault Symptom \\
\hline \hline$S_{1}$ & Condenser absolute pressure & $S_{9}$ & Condenser terminal temperature difference \\
\hline$S_{2}$ & Motor current of circulating water pump & $S_{10}$ & Super-cooling degree of condensate water \\
\hline$S_{3}$ & Circulating water pump inlet pressure & $S_{11}$ & Ejector and inlet temperature of cooling water \\
\hline$S_{4}$ & Condenser water resistance & $S_{12}$ & Pressure difference between the air from air \\
\hline$S_{5}$ & Condenser pump outlet pressure & $S_{13}$ & Water level of condenser \\
\hline$S_{6}$ & Motor current of condensate pump & $S_{14}$ & Motor current of vacuum pump \\
\hline$S_{7}$ & Condensate pump conductance & $S_{15}$ & Water level of LP heater \\
\hline$S_{8}$ & A rise of cooling water temperature & $S_{16}$ & Rotor differential expansion \\
\hline
\end{tabular}

\section{CONDENSER FAULT DIAGNOSIS BASED ON BP NEURAL NETWORK}

\subsection{The Model and Principle of BP Neural Network}

The basic idea of BP is that the learning process is composed of two process including forward diffusion of signal and backward diffusion of error. BP neural network is composed of three layers of neural network which are input layer, output layer and the hidden layer of the network [6]. The network between the upper and the lower layers of neurons are fully connected, but the mutual connection does not exist in the same layer. The neural network transition function adopted in this article is S function: $f(x)=1 /\left(1+\mathrm{e}^{-x}\right)$ [7].

Input of the input layer is $\boldsymbol{X}=\left(x_{0}, x_{1}, \ldots, x_{i}, \ldots, x_{n}\right)^{\mathrm{T}}$. Output of the hidden layer is $\boldsymbol{y}=\left(y_{0}, y_{1}, \ldots, y_{j}, \ldots, y_{m}\right)^{\mathrm{T}}$, and the output of the output layer is $\boldsymbol{O}=\left(\begin{array}{llll}o_{0}, & o_{1}, & o_{k}, & \ldots o_{l}\end{array}\right)^{\mathrm{T}}$. The expected output of the layer is $\boldsymbol{d}=\left(d_{1}, d_{2}, \ldots d_{k}, \ldots\right.$, $\left.d_{l}\right)^{\mathrm{T}}$. Where $x_{0}=y_{0}=-1$ is input layer and hidden layer threshold respectively. $\left(\boldsymbol{P}_{1}, \boldsymbol{P}_{2}, \ldots, \boldsymbol{P}_{j}, \ldots, \boldsymbol{P}_{m}\right),\left(\boldsymbol{Q}_{1}, \boldsymbol{Q}_{2}, \ldots\right.$, $\boldsymbol{Q}_{k}, \ldots, \boldsymbol{Q}_{l}$ ) are respectively the input layer to the hidden layer and the hidden layer to the output layer weights matrix, where the column vector of $\boldsymbol{P}_{j}$ is vector of hidden layer $j$ neurons, and $\boldsymbol{Q}_{k}$ is vector of output layer $k$ neurons corresponding [6].

The output of the hidden layer is $y_{j}=f\left(\right.$ net $\left._{j}\right)$, where net $_{j}=\sum_{i=0}^{n} P_{j} x_{i} \sum_{\mathrm{i}=0}^{\mathrm{n}} \mathrm{P}_{\mathrm{j}} \mathrm{x}_{\mathrm{i}}, j=1,2, \cdots, m$. The output of the output layer is $y_{j}=f\left(\right.$ net $\left._{j}\right)$, where net $_{k}=\sum_{j=0}^{m} Q_{k} y_{j}, \quad k=1$,

$2, \cdots, l$. The essence of BP algorithm is the forward propagation of signal and the back propagation of error. When the actual output and the expected output do not match, the definition of error should be:

$E=\frac{1}{2} \sum_{k=0}^{l}\left(d_{k}-o_{k}\right)^{2}$

The signal from the input layer transforms through hidden layer to output layer. If the output signal and the expected results do not match, it will be error back propagation step by step. According to constantly adjust network, until the error can reach the requirements [6].

\subsection{The Condenser Fault Diagnosis Based on BP Net- work}

Sixteen fault symptoms of condenser are selected as the input vector, which are defined as $\boldsymbol{S}\left(S_{1}, S_{2}, S_{3}, S_{4}, S_{5}, S_{6}, S_{7}\right.$,$\left.S_{8}, S_{9}, S_{10}, S_{11}, S_{12}, S_{13}, S_{14}, S_{15}, S_{16}\right)$ and shown in Table 1. Fourteen kinds of failure mode are selected as the output vector, which are defined as $\boldsymbol{F}\left(F_{1}, F_{2}, F_{3}, F_{4}, F_{5}, F_{6}, F_{7}, F_{8}, F_{9}, F_{10}\right.$,$\left.F_{11}, F_{12}, F_{13}, F_{14}\right)$ [7] and shown in Table 2 . Binary code is used for the elements in $\mathrm{F}$, which are represented as follows: F1(10000000000000), F2(01000000000000), F3(00100000000000), F4(00010000000000), F5(00001000000000), F6(00000100000000), F7(00000010000000), F8(00000001000000), F9(00000000100000), F10(00000000010000), F11(00000000001000), F12(00000000000100), F13(00000000000010), F14(00000000000001).

According to the condenser fault pattern knowledge base, the number of training samples is 14 groups, and the number of testing samples is 3 groups $\left(M_{1}, M_{2}, M_{3}\right)$. It calls the MATLAB neural network toolbox to create BP network to complete the training simulation. When the number of hidden layer neurons of BP network is 14, the effect of network training is best, and it is the best training accuracy after repeated testing. So the three layers neurons of BP network are $16,14,14$ respectively. The diagnosis results are shown in Table 3. The fault of testing sample $\left(M_{1}\right)$ is $F_{13}$ (circulating water shortage). The fault of testing sample $\left(M_{2}\right)$ is $F_{9}$ (cooling tube of condenser is choked). The fault of testing sample $\left(M_{3}\right)$ is $F_{4}$ (vacuum system pipe is broken). The results are completely consistent with the diagnosis results.

\section{CONDENSER FAULT DIAGNOSIS BASED ON PSO-BP NEURAL NETWORK}

\subsection{The Model and Principle of PSO-BP Neural Net- work}

The PSO-BP neural network is a learning algorithm based on the better combination of the global search ability of PSO algorithm and the local quick search ability of BP algorithm [9]. The basic idea of PSO-BP neural network is the combination of PSO algorithm and BP neural network 
Table 2. The condenser fault type set.

\begin{tabular}{|c|c|c|c|}
\hline Symbol & Fault Type & Symbol & Fault Type \\
\hline \hline$F_{1}$ & Circulating water pump failure & $F_{8}$ & Cooling water's Cooling tube is broken \\
\hline$F_{2}$ & Gas supply is suddenly interrupted & $F_{9}$ & Cooling tube of condenser is choked \\
\hline$F_{3}$ & The water in the condenser is overfull & $F_{10}$ & Condenser cooling tube is polluted \\
\hline$F_{4}$ & Vacuum system pipe is broken & $F_{11}$ & The air extractor does not working properly \\
\hline$F_{5}$ & Air cooling pipe plate dirty & $F_{12}$ & Vacuum system is not tight \\
\hline$F_{6}$ & Condensate pump fault & $F_{13}$ & Circulating water shortage \\
\hline$F_{7}$ & LP heater copper tube is broken & $F_{14}$ & Air ejector failure \\
\hline
\end{tabular}

Table 3. The test sample diagnosis based on BP neural network.

\begin{tabular}{|c|c|c|c|c|c|c|c|c|c|c|c|c|c|c|}
\hline $\mathbf{F}$ & $\mathbf{F}_{\mathbf{1}}$ & $\mathbf{F}_{\mathbf{2}}$ & $\mathbf{F}_{\mathbf{3}}$ & $\mathbf{F}_{\mathbf{4}}$ & $\mathbf{F}_{\mathbf{5}}$ & $\mathbf{F}_{\mathbf{6}}$ & $\mathbf{F}_{\mathbf{7}}$ & $\mathbf{F}_{\mathbf{8}}$ & $\mathbf{F}_{\mathbf{9}}$ & $\mathbf{F}_{\mathbf{1 0}}$ & $\mathbf{F}_{\mathbf{1 1}}$ & $\mathbf{F}_{\mathbf{1 2}}$ & $\mathbf{F}_{\mathbf{1 3}}$ & $\mathbf{F}_{\mathbf{1 4}}$ \\
\hline \hline $\mathrm{M}_{1}$ & 0.0055 & 0.0000 & 0.0031 & 0.0000 & 0.0080 & 0.0001 & 0.0000 & 0.0134 & 0.0170 & 0.0096 & 0.0193 & 0.0002 & 0.9893 & 0.0001 \\
\hline $\mathrm{M}_{2}$ & 0.0059 & 0.0000 & 0.0108 & 0.0006 & 0.0098 & 0.0001 & 0.0005 & 0.0037 & 0.9679 & 0.0036 & 0.0061 & 0.0001 & 0.0313 & 0.0000 \\
\hline $\mathrm{M}_{3}$ & 0.0038 & 0.0009 & 0.0004 & 0.9861 & 0.0000 & 0.0016 & 0.0003 & 0.0000 & 0.0001 & 0.0013 & 0.0004 & 0.0010 & 0.0000 & 0.0255 \\
\hline
\end{tabular}

based on the back-propagation training method of gradient descent. Initial weights are optimized by the PSO algorithm, and the optimal solution can be found in the smaller solution space by BP algorithm.

Then PSO-BP algorithm searches for the best initial weights and thresholds of BP neural network for the implementation of the process:

(1) PSO Variable Settings. A particle's position in the current population of BP neural network weights and threshold where is $X_{i}=\left[v_{11} \ldots v_{11} \ldots v_{N 1} \ldots v_{N 1} \ldots w_{11} \ldots w_{1 m} \ldots w_{11} \ldots w_{1 m}\right]^{\mathrm{T}}$ is set. The particle dimension is $D=l \times N+m \times l+l+m$. The current particle flying speed is $V_{i} . p b_{i}$ is expressed as the individual extreme and $g b_{i}$ is expressed as the global extreme [10]. The $i^{\text {th }}$ particle is to update its speed and position according to the following formula:

$V_{i d}{ }^{n+1}=w V_{\mathrm{id}}{ }^{\mathrm{n}}+C_{1} R_{1}\left(p b_{i d}{ }^{n}-X_{i d}{ }^{n}\right)+C_{2} R_{2}\left(g b_{i d}{ }^{n}-X_{i d}{ }^{n}\right)$

$X_{i d}{ }^{n+1}=X_{i d}{ }^{n}+V_{i d}{ }^{n+1}$

Where $w$ is the inertia weight. The first half of the whole has the higher global search ability to find the right seeds, and later has higher ability of development to accelerate the convergence speed. So the inertia weight value should be decreasing. It uses linearly decreasing weight strategy of the Eberhart:

$w(t)=\frac{\left(w_{\text {ini }}-w_{\text {end }}\right)\left(T_{\max }-t\right)}{T_{\max }}+w_{\text {end }}$

Where $t$ is the number of iterations, and $T_{\max }$ is the maximum number of iterations. $w_{i n i}$ as the initial value of inertia weight, also is the maximum value, and $w_{\text {end }}$ as at the end of iteration of the inertia weight value, is the minimum value.
(2) Fitness function and the terminating conditions. The updated particle position vector is putted into the neural network weights and thresholds, and their update the neural network [10]. The neural network takes training error as a function, namely fitness function of PSO:

$$
E_{p}=\frac{1}{2} \sum_{k=0}^{l-1}\left(d_{k}-Y_{k}\right)^{2}
$$

Where $d, Y$ are the expected output and the actual output of the $k^{\text {th }}$ sample input. When the evaluation function meets the accuracy requirement or reaches a maximum number of iterations to terminate the iteration loop $[12,13]$.

\subsection{Condenser Fault Diagnosis Based on PSO-BP Neural Network}

When it designs PSO-BP neural network, a three layer neural network is created. The numbers of three layers nodes are respectively $16,14,14$. The definition of particle swarm size is $40, c_{l}=c_{2}=1.45449$, and the maximum speed is $v_{\max }=0.5$. The maximum particle position is 1 , and the minimum is $-1, w_{\text {ini }}=0.95, w_{\text {end }}=0.40$, the largest number of iterations is $T_{\max }$, and the default error is $10^{-4}[5,16]$. Input vector is selected as the data in Table $\mathbf{1}$, and output vector is selected as the data in Table 2. According to the condenser fault symptom knowledge base, the number of training samples is 14 groups, and the number of testing samples is 3 groups $\left(M_{1}, M_{2}, M_{3}\right)$. It calls the MATLAB neural network toolbox to create three different kinds of PSO-BP neural network to complete the simulation training. The basic idea of the basic PSO-BP algorithm is inertia weight using linearly decreasing weight strategy of the Eberhart. The core idea of improved PSO-BP (1) is the improved learning factor. The basic idea 
Table 4. The test sample diagnosis based on PSO-BP neural network.

\begin{tabular}{|c|c|c|c|c|c|c|c|c|c|}
\hline \multirow{2}{*}{$\begin{array}{c}\text { Algorithm } \\
\text { Sample }\end{array}$} & \multicolumn{3}{|c|}{ The Basic PSO-BP } & \multicolumn{3}{|c|}{ Improved PSO-BP(1) } & \multicolumn{3}{|c|}{ Improved PSO-BP(2) } \\
\hline & $M_{1}$ & $M_{2}$ & $M_{3}$ & $M_{1}$ & $M_{2}$ & $M_{3}$ & $M_{1}$ & $M_{2}$ & $M_{3}$ \\
\hline$F_{2}$ & 0.0002 & 0.0001 & 0.0005 & 0.0012 & 0.0006 & 0.0013 & 0.0001 & 0.0000 & 0.0007 \\
\hline$F_{3}$ & 0.0001 & 0.0029 & 0.0000 & 0.0012 & 0.0006 & 0.0000 & 0.0015 & 0.0007 & 0.0000 \\
\hline$F_{5}$ & 0.0025 & 0.0018 & 0.0000 & 0.0248 & 0.0013 & 0.0001 & 0.0105 & 0.0040 & 0.0000 \\
\hline$F_{6}$ & 0.0000 & 0.0007 & 0.0001 & 0.0048 & 0.0041 & 0.0002 & 0.0035 & 0.0003 & 0.0014 \\
\hline$F_{7}$ & 0.0001 & 0.0003 & 0.0001 & 0.0000 & 0.0004 & 0.0004 & 0.0000 & 0.0041 & 0.0002 \\
\hline$F_{10}$ & 0.0009 & 0.0000 & 0.0000 & 0.0103 & 0.0010 & 0.0217 & 0.0037 & 0.0018 & 0.0045 \\
\hline$F_{11}$ & 0.0022 & 0.0123 & 0.0000 & 0.0025 & 0.0098 & 0.0006 & 0.0001 & 0.0003 & 0.0004 \\
\hline$F_{12}$ & 0.0000 & 0.0000 & 0.0036 & 0.0000 & 0.0001 & 0.0657 & 0.0000 & 0.0031 & 0.0118 \\
\hline$F_{13}$ & 0.9979 & 0.0122 & 0.0000 & 0.9759 & 0.0186 & 0.0000 & 0.9830 & 0.0156 & 0.0000 \\
\hline$F_{14}$ & 0.0000 & 0.0000 & 0.0025 & 0.0001 & 0.0000 & 0.0154 & 0.0000 & 0.0000 & 0.0422 \\
\hline Time & \multicolumn{3}{|c|}{$16.08 \mathrm{~s}$} & \multicolumn{3}{|c|}{$13.58 \mathrm{~s}$} & \multicolumn{3}{|c|}{$12.04 \mathrm{~s}$} \\
\hline
\end{tabular}

of improved PSO-BP (2) is inertia weight using nonlinear decreasing inertia weight strategy. The diagnosis results as shown in Table 4. The fault of testing sample $\left(M_{1}\right)$ is $F_{13}$ (circulating water shortage). The fault of testing sample $\left(M_{2}\right)$ is $F_{9}$ (cooling tube of condenser is choked). The fault of testing sample $\left(M_{3}\right)$ is $F_{4}$ (vacuum system pipe is broken). The results are completely consistent with the diagnosis results. The basic PSO-BP network, the improved PSO-BP network (1) and improved PSO-BP neural network (2) are not much difference in the identification of samples, but the improvement of PSO-BP network (2) in the convergence rate has a little faster than the basic PSO-BP neural network and improved PSO-BP neural network (1).

\section{CONDENSER FAULT DIAGNOSIS BASED ON SUPPORT VECTOR MACHINE}

\subsection{The Model and Principle of Support Vector Machine}

SVM is the development of optimal classification surface under linearly separable case [11]. It is assumed that there is the given training set, $\left\{\left(x_{1}, y_{1}\right),\left(x_{2}, y_{2}\right), \ldots,\left(x_{n}, y_{n}\right)\right\}$, where is $x_{i} \in R_{n}, y \in\{1,-1\}$. Supposing all the vector of the training set can be a hyper-plane $(\omega \cdot x)+b=0$ linear division. The distance of the heterogeneous vector closest to the hyper-plane is far, and the hyper-plane is the optimal hyperplane. The distance over heterogeneous vector plane recently called support vector. A set of support vectors can be determined uniquely a hyper-plane [5].
For the linearly separable problems, it can be assumed that the vector in the training set to meet as follow:

$y_{1}\left(\omega \cdot x_{i}+b\right) \geq 1$

The problem of the constructing optimal hyper-plane is converted into under the constraint of type (6) seeking the minimum value of a type:

$\phi(\omega)=\frac{1}{2}\|\omega\|^{2}$

The optimal solution of the optimization problem is the saddle point of the following Lagrange function:

$L(\omega, b, \alpha)=\frac{1}{2}\|\omega\|^{2}-\sum_{i=1}^{n} \alpha_{1}\left[y_{i}(\omega \cdot x+b)-1\right]$

Where $\alpha$ is the Lagrange multiplier.

The basic idea of nonlinear SVM is that the input vector $\boldsymbol{x}$ is mapped into a high dimensional feature space by a nonlinear mapping determined in advance, and then build the optimal hyper-plane in a high dimensional space. Because of the vector just carrying out dot product operation, if it uses the kernel function, it can avoid the complex operation in the high dimensional feature space $[14,15]$. The kernel function $K$ satisfies:

$$
K\left(x_{i}, x_{j}\right)=\psi\left(x_{i}\right) \cdot \psi\left(x_{j}\right)
$$

The objective function of quadratic programming problem becomes: 


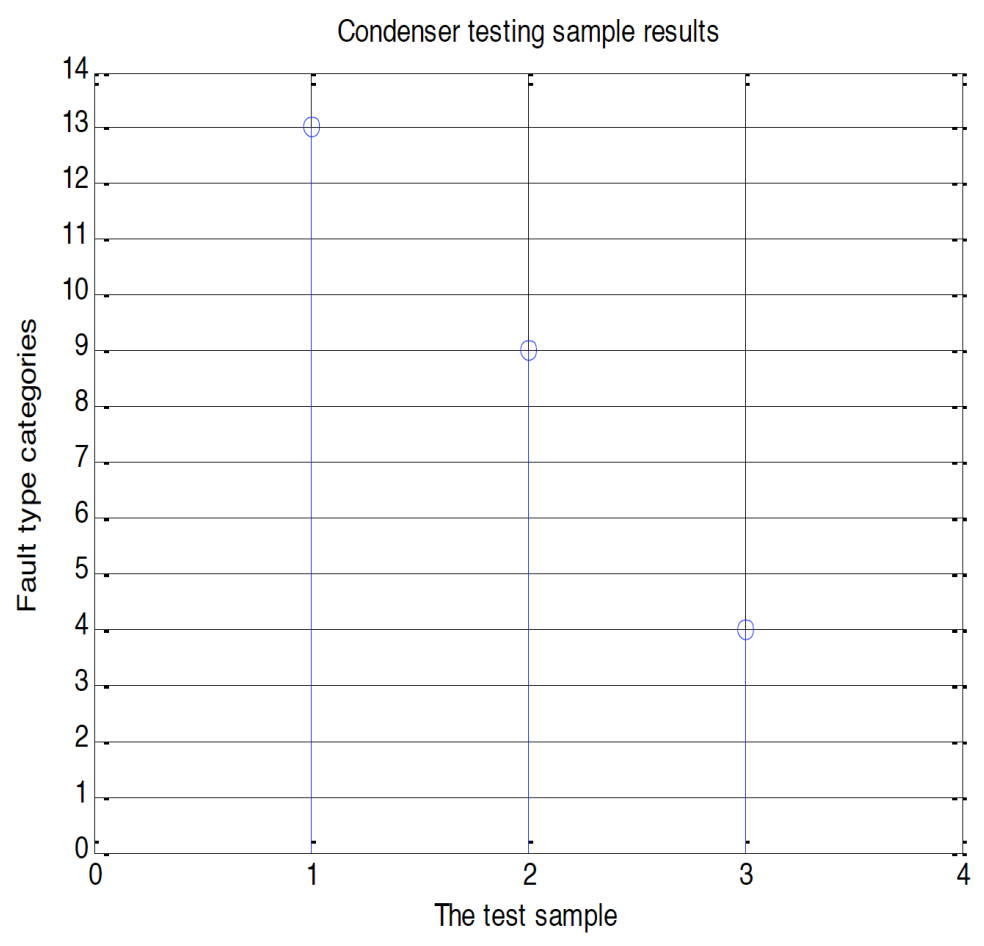

Fig. (1). The test results based on SVM diagram.

$W(\alpha)=\sum_{i=1}^{n} \alpha_{i}-\frac{1}{2} \sum_{i, j}^{n} \alpha_{i} \alpha_{j} y_{i} y_{j} K\left(x_{i}, x_{j}\right)$

After training, the following function symbols are calculated:

$$
f(x)=\sum_{S V} \alpha_{i} y_{j} K\left(x_{i}, x\right)+b
$$

\subsection{Condenser Fault Diagnosis Based on Support Vector Machine}

It adds a toolbox of lib-svm into MATLAB to create the SVM training simulation. Input vector is selected as the data in Table 1, and output vector is selected as the data in Table $\mathbf{2}$. According to the condenser fault symptom knowledge base, the number of training samples is 14 groups, and the number of testing samples is 3 groups $\left(M_{1}, M_{2}, M_{3}\right)$. This article calls the MATLAB neural network toolbox to create SVM simulation training. The diagnosis results as shown in Fig. (1). The fault of testing sample $\left(M_{1}\right)$ is $F_{13}$ (circulating water shortage). The fault of testing sample $\left(M_{2}\right)$ is $F_{9}$ (cooling tube of condenser is choked). The fault of testing sample $\left(M_{3}\right)$ is $F_{4}$ (vacuum system pipe is broken). The results are completely consistent with the diagnosis results.

\section{THE COMPARISON OF THREE DIFFERENT AL- GORITHMS}

Three different algorithms, the BP neural network, PSOBP neural network and SVM, were used in this article for condenser fault diagnosis. Although the number of training samples was not large, the highest recognition ratio of the 30 groups of testing samples still can be up to $76.67 \%, 95.56 \%$ and $100 \%$ (Results in Table 5, which proved that three different algorithms, with strong nonlinear classification ability and generalization ability, had a high reliability and practicality in condenser fault diagnosis).

Through Table 5 and Table 6, compared with three different algorithms, the following conclusions were drawn:

BP neural network has strong nonlinear mapping ability, adaptive ability and generalization ability. The recognition ratio of $\mathrm{BP}$ is $76.67 \%$. The convergence speed of BP neural network has a little faster. Its training error could limit to $10^{-4}$.

Compared with the BP neural network, PSO-BP neural network overcome the slow convergence speed and easily fall into local extreme limitations. The recognition ratio of the basic PSO-BP, improved PSO-BP(1) and improved PSO$\mathrm{BP}(2)$ are respectively $90 \%, 96.67 \%, 100 \%$. The average recognition ratio of $\mathrm{PSO}-\mathrm{BP}$ is $95.56 \%$. The precision of the model is better, and PSO-BP well improved learning ability and generalization ability of BP network. The training epoch of PSO-BP neural network is shorter than BP neural network. Its training error could limit to $10^{-4}$.

SVM is better than the previous two kinds of neural network in the training error. The recognition ratio of SVM is $100 \%$. Although the recognition ratio of improved PSO$\mathrm{BP}(2)$ is $100 \%$, training time of improved PSO-BP(2) is longer than training time of SVM. The classification ability of SVM is more accurate, more suitable for the processing pattern classification problems. The SVM does not require an iterative training during the simulation. The speed classification of SVM in three kinds of networks is the fastest. In addition, the theoretical accumulation of fault samples can make the SVM expanding to further improve the fault identification, and thus SVM has a certain extrapolation extension performance. 
Table 5. The comparison of three different algorithms to test sample recognition rate.

\begin{tabular}{|c|c|c|c|}
\hline Algorithms & Sample & Recognition Rate & The Average Recognition Rate \\
\hline \multirow{3}{*}{ BP } & $M_{1}$ & $80 \%$ & \multirow{3}{*}{$76.67 \%$} \\
\hline & $M_{2}$ & $80 \%$ & \\
\hline & $M_{3}$ & $70 \%$ & \\
\hline \multirow{3}{*}{ The basic PSO-BP } & $M_{1}$ & $100 \%$ & \multirow{3}{*}{$90 \%$} \\
\hline & $M_{2}$ & $80 \%$ & \\
\hline & $M_{3}$ & $90 \%$ & \\
\hline \multirow{3}{*}{ Improved PSO-BP(1) } & $M_{1}$ & $90 \%$ & \multirow{3}{*}{$96.67 \%$} \\
\hline & $M_{2}$ & $100 \%$ & \\
\hline & $M_{3}$ & $100 \%$ & \\
\hline \multirow{3}{*}{ Improved PSO-BP(2) } & $M_{1}$ & $100 \%$ & \multirow{3}{*}{$100 \%$} \\
\hline & $M_{2}$ & $100 \%$ & \\
\hline & $M_{3}$ & $100 \%$ & \\
\hline \multirow{3}{*}{ SVM } & $M_{1}$ & $100 \%$ & \multirow{3}{*}{$100 \%$} \\
\hline & $M_{2}$ & $100 \%$ & \\
\hline & $M_{3}$ & $100 \%$ & \\
\hline
\end{tabular}

Table 6. Three different algorithms to diagnosis the condenser fault training process.

\begin{tabular}{|c|c|c|c|}
\hline Network Type & Training Time (s) & Training Epoch & Training Error \\
\hline \hline BP & 15.72 & 2000 & 0.0001 \\
\hline The basic PSO-BP & 16.08 & 30 & 0.0001 \\
\hline Improved PSO-BP(1) & 13.58 & 30 & 0.0001 \\
\hline Improved PSO-BP(2) & 12.04 & 30 & 0.0001 \\
\hline SVM & 1.977 & - & 0 \\
\hline
\end{tabular}

\section{CONCLUSION}

The identification task of condenser fault diagnosis can be achieved by BP neural network, PSO-BP neural network and SVM. Although the recognition ratio of improved PSO$\mathrm{BP}(2)$ is $100 \%$, training time of improved PSO-BP(2) is longer than training time of SVM. And the performance of SVM is the best method among three different algorithms. SVM has a good rapidity. The calculation results of the SVM method, under the condition of small samples, is better than the calculation results of the other two methods. The generalization ability of SVM is stronger, and the efficiency ability of SVM is higher than those of the neural network. Therefore, the method based on SVM is able to adapt to the rapidity and accuracy requirement of condenser fault diagnosis, and is easy to be applied to the diagnosis. SVM algorithm has a broad application prospect. But for a large number of training samples SVM algorithm is difficult to implement. When the number of the sample is large, storage and calculation of the matrix will cost a lot of machine memory and computing time. Using SVM to solve the multi-class problem is difficult. SVM for other fault diagnosis needs further research and improvement.

\section{CONFLICT OF INTEREST}

The author confirms that this article content has no conflict of interest.

\section{ACKNOWLEDGEMENTS}

This work was supported by the State Key Program of National Natural Science Foundation of China (Grant No. 61034004), Shanghai Science and Technology Commission Key Program (Grant No. 13111104300) and Shanghai Key Laboratory of Power Station Automation Technology (Grant No. 13DZ2273800). 


\section{REFERENCES}

[1] F. Xia, H. Zhang, D. Peng, H. Li and L. Xu, "Research of the reliability coefficient in information fusion", 2009 International Conference on Signal Acquisition and Processing, Kuala Lumpur, 2009, pp.85-88.

[2] L. Wang, R. Zhang, W. Sheng, and Z. Xu, "Condenser fault diagnosis based on fuzzy rules and support vector machine", J. Eng. Therm. Energy Power, vol. 24, pp. 476-480, 2009.

[3] M. Geethanjali, and S. M. R. Slcohanal, "PSO Trained ANN-based differential protection scheme for power transformers", Original Research Article Neuraocomputing, vol. 71, pp. 904-918, 2008.

[4] K. Salahshoor, M. Kordestani, and M. S. Khoshro, "Fault detection and diagnosis of an industrial steam turbine using fusion of SVM and ANFIS classifiers", Original Research Article Energy, vol. 35, pp. 5472-5482, 2010.

[5] H. Gong, "Study of fault diagnosis for condenser based on support vector machine", Thermal Turbine, vol.38, pp.57-60, 2009.

[6] L. Cao, R. Li, Y. Yuan, M. Li, and X. Chen, "Study on the method of map evaluation based on bp network", Packaging Engineering, vol. 35, pp. 38-41, 2009.

[7] L. Zhang, W. Wang, and D. Tang, "The improved BP network application in the condenser fault diagnosis", Thermal Power Generation, vol. 3, pp. 50-52, 2004.

[8] M. Demetgul, I. N. Tansel, and S. Taskin, "Fault diagnosis of pneumatic systems with artificial neural network algorithms",
Original Research Article Expert Systems With Applications, vol. 36, pp. 10512-10519, 2009.

[9] H. Feng, X. Wu, R. Yuan and L. Su, "Fault diagnosis of engine based on pso-bp neural network", Control and Instruments in Chemical Industry, vol. 1, pp. 76-79, 2009.

[10] S. Zhang, "Algorithm of short-time load forecast based on PSO-BP neural network", Modern Electronics Technique, vol. 36, pp. 155$158,2013$.

[11] K. Chen, L. Chen, M. Chen and C. Lee. "Using SVM based method for equipment fault detection in a thermal power plant", Computers in Industry, vol. 62, pp. 42-50, 2011.

[12] L. Ma, B. Wang, Z. Tong, Yongguang Ma and ZhipingSong, "Fuzzy pattern recognition and artificial neural network used for fault diagnosis of the double-channel condenser", Proceedings of the CSEE, vol. 21, pp. 69-74, 2001.

[13] L. Li, and B. Niu, Particle Swarm Optimization Algorithm, Beijing: Metallurgical Industry Press, 2009.

[14] V. Muralidharan, V. Sugumaran, and V. Indira, "Fault diagnosis of monoblock centrifugal pump using SVM", Engineering Science and Technology, vol. 4, pp. 1-6, 2014.

[15] J. Zhou, P. Yang, and N. Yang, "Comparison and application of bp and elman network in fault diagnosis of condenser", East China Electric Power, vol. 39, pp. 822-825, 2011.

[16] Y. Tian, Hybrid Neural Network Technology, Beijing: Science Press, 2009.

Received: October 29, 2014

Revised: November 02, 2014

Accepted: November 11, 2014

(C) Fei et al.; Licensee Bentham Open.

This is an open access article licensed under the terms of the Creative Commons Attribution Non-Commercial License (http://creativecommons.org/licenses/by-nc/3.0/) which permits unrestricted, non-commercial use, distribution and reproduction in any medium, provided the work is properly cited. 